\title{
The existence of infinitely many bifurcating branches
}

\author{
Hans-Jörg Ruppen \\ Niedergampelstrasse, 3945 Gampel, Switzerland
}

(MS received 25 May 1984. Revised MS received 8 November 1984)

\section{Synopsis}

We consider the non-linear problem $-\Delta u(x)-f(x, u(x))=\lambda u(x)$ for $x \in \mathbb{R}^{N}$ and $u \in W^{1,2}\left(\mathbb{R}^{N}\right)$. We show that, under suitable conditions on $f$, there exist infinitely many branches all bifurcating from the lowest point of the continuous spectrum $\lambda=0$. The method used in the proof is based on a theorem of Ljusternik-Schnirelman type for the free case.

\section{Introduction}

We consider the following non-linear problem:

$$
-\Delta u(x)-f(x, u(x))=\lambda u(x) \text { for } x \in \mathbb{R}^{N} .
$$

This problem has been treated by many authors including Berger, Strauss, Berestycki and Lions. In this paper we follow Stuart $[\mathbf{4}, \mathbf{5}]$.

We prove the following theorem.

THEOREM 1.1. Suppose $f(x, u(x))=q(x)|u(x)|^{\sigma} u(x)$ where $q \in L^{p}\left(\mathbb{R}^{N}\right)$ with $\max \{N / 2,2\} \leqq p \leqq \infty, 0<\sigma<2(2-N / p) /(N-2)$, and $q(x)>0$ for almost all $x \in \mathbb{R}^{N}$.

Suppose further that there exist constants $A, t>0$ such that $q(x) \geqq A /(1+|x|)^{t}$ for almost all $x \in \mathbb{R}^{N}$ and that $0<t<2-N \sigma / 2$.

Then,

(i) for $\lambda<0$, the equation

$$
-\Delta u(x)-q(x)|u(x)|^{\sigma} u(x)=\lambda u(x) \quad \text { for } x \in \mathbb{R}^{N}
$$

has infinitely many distinct pairs of (generalised) solutions $\left\{\left(\lambda, \pm u_{k}^{\lambda}\right)\right\}_{k \in \mathbb{N}}$;

(ii) the lowest point of the continuous spectrum is a bifurcation point; in fact all solutions $\left(\lambda, \pm u_{k}^{\lambda}\right)$ bifurcate from $\lambda=0$ :

$$
\left\|u_{k}^{\lambda}\right\|_{T} \rightarrow 0 \text { as } \lambda \rightarrow 0^{-} \text {. }
$$

We prove this theorem even for more generalised non-linearities $f(x, u(x))$ such as used by Stuart [4]. (See Conditions $\left(\mathrm{A} 1^{*}\right),\left(\mathrm{A} 2^{*}\right),\left(\mathrm{A} 3^{*}\right)$ and (A4*) below.)

The existence of an infinite number of solutions for each negative value of $\lambda$ has been established by Berestycki and Lions [2], at least when $f(x, u(x))=$ $\mathrm{g}(u(x))$. Stuart has shown that $\lambda=0$ is a bifurcation point and that there exists a branch of solutions bifurcating from $\lambda=0[4,5]$. What we show is that in fact there exist infinitely many branches all bifurcating from the lowest point of continuous spectrum $\lambda=0$. 
The main tool is a generalised result of Ambrosetti and Rabinowitz $[\mathbf{1}, \mathbf{3}]$ concerning the existence of an infinite number of critical points of a functional. It involves the investigation of the functional on sets of arbitrary genus and we construct such sets using functions of the following type:

$$
u(x)=p\left(|x|^{2}\right) e^{-|x|^{2}} \text { for } x \in \mathbb{R}^{N}
$$

where $p$ is a polynomial. This construction seems simpler than that used previously for problems of this kind [2].

An alternative approach is discussed in Section 6.

\section{The equation $T^{\prime} T u-F(u)=\lambda u$}

We consider the equation

$$
-\Delta u(x)-f(x, u(x))=\lambda u(x), \quad x \in \mathbb{R}^{N}, \quad N \geqq 2
$$

and the corresponding bifurcation problem, but first we give a precise meaning to this equation. (This section follows Stuart [4].)

Let us begin with the operator $-\Delta$.

We put

$$
\begin{gathered}
H:=L^{2}\left(\mathbb{R}^{N}\right)=L^{2}, \quad\|u\|:=\left\{\int u(x)^{2} d x\right\}^{\frac{1}{2}}, \\
\mathscr{D}(S):=\left\{u \in H: \sum_{i=1}^{N} D_{i}^{2} u \in H\right\}, \quad S u:=-\sum_{i=1}^{N} D_{i}^{2} u,
\end{gathered}
$$

i.e. $S$ is the self-adjoint extension of the negative Laplacian in $H$. When no domain of integration is indicated, it is understood that the integration is over all of $\mathbb{R}^{N}$. Let $\left(H_{2},\|.\|_{2}\right)$ be the Hilbert space obtained by equipping $\mathscr{D}(S)$ with the graph norm

$$
\|u\|_{2}:=\left\{\|u\|^{2}+\|S u\|^{2}\right\}^{\frac{1}{2}}, \quad \forall u \in \mathscr{D}(S) .
$$

Then, up to equivalence of norms, $H_{2}=W^{2,2}\left(\mathbb{R}^{N}\right)$.

We now take $T=S^{\frac{1}{2}}$, the positive self-adjoint square root of $S$. Let $\left(H_{T},\|.\|_{T}\right)$ be the Hilbert space obtained by equipping $\mathscr{D}(T)$ with the graph norm

$$
\|u\|_{T}:=\left\{\|u\|^{2}+\|T u\|^{2}\right\}^{\frac{1}{2}}, \quad \forall u \in \mathscr{D}(T) .
$$

Then $H_{T}=W^{2,1}\left(\mathbb{R}^{N}\right)$ and $\|T u\|=\|\nabla u\|, \forall u \in H_{\mathrm{T}}$ where $\nabla u=\left(D_{1} u, \ldots, D_{\mathrm{N}} u\right)$.

By identifying $H$ with $H^{*}$, we can write $H_{\mathrm{T}} \subset H=H^{*} \subset\left(H_{\mathrm{T}}\right)^{*}$ and use $\langle.,$.$\rangle for$ the duality between $\left(H_{\mathrm{T}}\right)^{*}$ and $H_{\mathrm{T}}$. Since $T: H_{\mathrm{T}} \rightarrow H$ is bounded, it has a conjugate $T^{\prime}: H^{*}=H \rightarrow\left(H_{\mathrm{T}}\right)^{*}$ which is also bounded. Then $T^{\prime} T: H_{T} \rightarrow\left(H_{\mathrm{T}}\right)^{*}$ is a bounded linear operator such that $T^{\prime} T u=S u, \forall u \in \mathscr{D}(S)$ and $\mathscr{D}(S)=$ $\left\{u \in H_{T}: T^{\prime} T u \in H\right\}$.

These results are discussed in more detail in [4].

We now turn to $f$ in (2.1) and make the following basic assumption.

(A1) The function $f$ can be written as a sum, $f=\sum_{i=1}^{m} f_{i}$, of a finite number of functions $f_{i}$ where, for $1 \leqq i \leqq m, f_{i}: \mathbb{R}^{N} \times \mathbb{R} \rightarrow \mathbb{R}$ is of Carathéodory type such 
that

$$
\left|f_{i}(x, s)\right| \leqq A_{i}(x)|s|^{1+\sigma_{i}}
$$

for all $s \in \mathbb{R}$ and almost all $x \in \mathbb{R}^{N}$, where $A_{i} \in L^{p_{i}}\left(\mathbb{R}^{N}\right)$ for some $p_{i}$ such that $\max \{N / 2,2\} \leqq p_{i} \leqq \infty$ and $0<\sigma_{i}<2\left(2-N / p_{i}\right) /(N-2)$.

(When $p=\infty, 1 / p$ is understood to be 0 and when $N=2,1 /(N-2)$ is understood to be $+\infty$.)

This assumption guarantees a well-posed problem (2.1) in the sense that

$$
f(x, u(x)) \in\left(H_{\mathrm{T}}\right)^{*} \quad \text { whenever } u \in H_{\mathrm{T}} \text {. }
$$

In fact, if (A1) is satisfied, we set

$$
\mathscr{F}_{i}(x, s):=\int_{0}^{s} f_{i}(x, r) d r \quad \text { and } \quad \mathscr{F}:=\sum_{i=1}^{m} \mathscr{F}_{i} .
$$

For $u: \mathbb{R}^{N} \rightarrow \mathbb{R}$, let

$$
\begin{gathered}
F_{i}(u)(x):=f_{i}(x, u(x)) \quad \text { and } \quad F:=\sum_{i=1}^{m} F_{i}, \\
\varphi_{i}(u):=\int \mathscr{F}_{i}(x, u(x)) d x \quad \text { and } \quad \varphi:=\sum_{i=1}^{m} \varphi_{i} .
\end{gathered}
$$

The problem (2.1) is then equivalent to

$$
S u-F(u)=\lambda u, \quad u \in H_{2}
$$

The following result is also given by Stuart [4].

Proposition 2.1. Let Condition (A1) hold.

(i) For $1 \leqq i \leqq m, F_{i}$ maps $L^{\tau_{i}}$ boundedly and continuously to $L^{q_{i}}$ where

$$
\tau_{i}:=\left(2+\sigma_{i}\right) /\left(1-1 / p_{i}\right) \text { and } q_{i}:=\tau_{i} /\left(\tau_{i}-1\right) \text {, }
$$

and $F$ maps $H_{T}$ boundedly and continuously to $\left(H_{\mathrm{T}}\right)^{*}$. Further,

$$
\left|\left\langle F_{i}(u), u\right\rangle\right| \leqq K_{i}\|T u\|^{\alpha_{i}}\|u\|^{\beta_{i}}, \quad \forall u \in H_{\mathrm{T}},
$$

where $\alpha_{i}:=N\left(\sigma_{i} / 2+1 / p_{i}\right), \beta_{i}:=2+\sigma_{i}-\alpha_{i}$ and $K_{i}>0$ and so

$$
|\langle F(u), u\rangle| \leqq \max _{1 \leqq i \leqq m}\left\{m K_{i}\|T u\|^{\alpha_{i}}\|u\|^{\beta_{i}}\right\}, \quad \forall u \in H_{T}
$$

(ii) If in addition to Assumption (A1) we have

$$
0<\sigma_{i}<2\left(1-N / p_{i}\right) /(N-2) \text { for } 1 \leqq i \leqq m,
$$

then $F_{i}$ maps $L^{\tau_{i}}$ boundedly and continuously into $H$.

(iii) For $1 \leqq i \leqq m, \varphi: H_{T} \rightarrow \mathbb{R}$ is continuously Fréchet differentiable and $\varphi^{\prime}(u) v=$ $\langle F(u), v\rangle$ for all $u, v \in H_{\mathrm{T}}$.

Remark. We note that $2<\tau_{i}<2 N /(N-2)$ and so $H_{\mathrm{T}}$ is continuously embedded in $L^{\tau_{i}}$ for $1 \leqq i \leqq m$ by the Sobolev embedding. It follows that $L^{q_{i}}$ is continuously embedded in $\left(H_{\mathrm{T}}\right)^{*}$. 
A pair $(\lambda, u)$ is now called a (generalised) solution of (2.2) if

(i) $(\lambda, u) \in \mathbb{R} \times H_{T}$

and

(ii) $T^{\prime} T u-F(u)=\lambda u$ holds in $\left(H_{T}\right)^{*}$, i.e.

$$
\left\langle T^{\prime} T u, v\right\rangle-\langle F(u), v\rangle=\lambda\langle u, v\rangle
$$

for all $v \in H_{T} . \lambda$ is called a $\left(L^{2}-\right)$ bifurcation point for (2.2) if there exists a sequence $\left\{\left(\lambda_{n}, u_{n}\right)\right\}$ of (generalised) solutions to (2.2) such that

$$
\begin{gathered}
u_{n} \neq 0, \quad \forall n \in \mathbb{N}, \\
\lambda_{n} \rightarrow \lambda \text { and }\left\|u_{n}\right\|_{T} \rightarrow 0 \text { for } n \rightarrow \infty .
\end{gathered}
$$

We want to show that $\lambda=0$ is a bifurcation point for (2.2) and that there exist infinitely many bifurcating branches; to do this we need $\varphi$ to be weakly sequentially continuous. This is guaranteed by the following assumption.

(A1*) $f$ satisfies Condition (A1) and $A_{i}(x) \rightarrow 0$ for $|x| \rightarrow \infty$ whenever $p_{i}=\infty$ and $f(x, s)$ is odd with respect to $s$.

Then the following result holds.

Proposition 2.2. Let Condition (A1*) hold. Then $F$ is completely continuous and compact; more precisely

$$
u_{n} \rightarrow u \text { in } H_{\mathrm{T}} \Rightarrow F\left(u_{n}\right) \rightarrow F(u) \text { in }\left(H_{\mathrm{T}}\right)^{*} \text { for } n \rightarrow \infty .
$$

Corollary 2.3. Let Condition (A $1^{*}$ ) hold. Then $\varphi: H_{T} \rightarrow \mathbb{R}$ is weakly sequentially continuous.

Proof. This follows from the compactness of $\varphi^{\prime}=F$. See [6, Satz 39.22].

\section{The functional $\boldsymbol{J}_{\boldsymbol{\lambda}}$}

For $\lambda<0$, we put

$$
\|u\|_{\lambda}=\left\{\|T u\|^{2}-\lambda\|u\|^{2}\right\}^{\frac{1}{2}}, \quad \forall u \in H_{\mathrm{T}} .
$$

Note that $\|\cdot\| \|_{\lambda}$ and $\|\cdot\|_{T}$ are equivalent norms in $H_{T}$. We now define a functional $J_{\lambda}$ whose critical points are (generalised) solutions to (2.1); critical points in turn will be found via a theorem of Ljusternik-Schnirelman type for the free case.

We put

$$
J_{\lambda}: H_{T} \rightarrow \mathbb{R}, \quad u \mapsto J_{\lambda}(u):=\frac{1}{2}\|u\|_{\lambda}^{2}-\varphi(u) .
$$

In order to control the radial behaviour of $J_{\lambda}$, we make the following assumption on $f$.

(A2*) There exist constants $\bar{\sigma} \geqq \underline{\sigma}>0$ such that for every $s \in \mathbb{R}$

$$
\mathscr{F}(x, t s) \geqq t^{2+\Phi \mathscr{F}}(x, s) \geqq 0 \quad \text { whenever } t \geqq 1
$$

and

$$
\mathscr{F}(x, t s) \geqq t^{2+\tilde{\sigma}} \mathscr{F}(x, s) \geqq 0 \quad \text { whenever } 0 \leqq t \leqq 1 .
$$


The existence of infinitely many bifurcating branches

Further,

$$
\varphi(u)>0 \text { for all } u \in H_{T} \backslash\{0\} .
$$

Note that Condition (A2*) will be satisfied if the function $f$ is of the form

$$
f(x, s)=\sum_{i=1}^{m} q_{i}(x)|s|^{\sigma_{i}} s
$$

with $q_{i}(x)>0$ for almost all $x \in \mathbb{R}^{N}$ and $\sigma_{i}>0$. (Simply take $g:=\min \left\{\sigma_{i}: 1 \leqq i \leqq m\right\}$, $\bar{\sigma}:=\max \left\{\sigma_{i}: 1 \leqq i \leqq m\right\}$.)

In order to have a quantitative control on the radial behaviour of $J_{\lambda}$, we introduce a second functional on $H_{\mathrm{T}}$ :

where

$$
I_{\lambda}: H_{T} \rightarrow \mathbb{R}, u \mapsto I_{\lambda}(u)= \begin{cases}0 & (u=0), \\ \frac{1}{2}\|u\|_{\lambda}^{2}-\|u\|_{\lambda}^{2+\sigma} \psi(u) & \left(u \in H_{T},\|u\| \|_{\lambda} \geqq 1\right), \\ \frac{1}{2}\|u\|_{\lambda}^{2}-\|u\|_{\lambda}^{2+\bar{\sigma}} \psi(u) & \left(u \in H_{T}, 0<\|u\|_{\lambda} \leqq 1\right),\end{cases}
$$

$$
\psi(u):=\varphi\left(u /\|u\|_{\lambda}\right) \text { for } u \in H_{T} \backslash\{0\} .
$$

Then $I_{\lambda}$ is a majorant functional for $J_{\lambda}$ whose radial behaviour can be completely controlled. In fact, the following lemma follows immediately from the definition of $I_{\lambda}$ and Condition (A2*).

LEMMA 3.1. Let Condition (A2*) be satisfied. Then

$$
\begin{array}{ll}
J_{\lambda}(u)=I_{\lambda}(u) & \text { whenever } \quad\|u\|_{\lambda}=1 . \\
J_{\lambda}(u) \leqq I_{\lambda}(u) & \text { for all } u \in H_{\mathrm{T}} .
\end{array}
$$

Let us now have a look at the radial behaviour of $I_{\lambda}$. For any fixed $u \in H_{\mathrm{T}} \backslash\{0\}$, we put

i.e.

$$
a:[0, \infty) \rightarrow \mathbb{R}, \quad t \mapsto a(t):=I_{\lambda}(t u),
$$

$$
a(t)= \begin{cases}0 & (t=0), \\ \frac{1}{2} t^{2}\|u\|_{\lambda}^{2}-t^{2+\bar{\sigma}}\|u\|_{\lambda}^{2+\bar{\sigma}} \psi(u) & \left(0<t \leqq 1 /\|u\|_{\lambda}\right), \\ \frac{1}{2} t^{2}\|u\|_{\lambda}^{2}-t^{2+g}\|u\|_{\lambda}^{2+\sigma} \psi(u) & \left(t \geqq 1 /\|u\|_{\lambda}\right) .\end{cases}
$$

Then

$$
a^{\prime}(t)= \begin{cases}0 & (t=0), \\ t\|u\|_{\lambda}^{2}\left\{1-(2+\bar{\sigma}) t^{\tilde{\sigma}}\|u\| \|_{\lambda}^{\bar{\sigma}} \psi(u)\right\} & \left(0<t<1 /\|u\|_{\lambda}\right), \\ t\|u\|_{\lambda}^{2}\left\{1-(2+\underline{\sigma}) t^{\sigma}\|u\| \|_{\lambda}^{\underline{\sigma}} \psi(u)\right\} & \left(t>1 /\|u\|_{\lambda}\right),\end{cases}
$$

and

$$
\begin{aligned}
& a_{-}^{\prime}\left(1 /\|u\|_{\lambda}\right)=\|\| u \|_{\lambda}\{1-(2+\bar{\sigma}) \psi(u)\}, \\
& a_{+}^{\prime}\left(1 /\|u\|_{\lambda}\right)=\|u\|_{\lambda}\{1-(2+\underline{\sigma}) \psi(u)\},
\end{aligned}
$$

where $a_{-}^{\prime}$ and $a_{+}^{\prime}$ are the left and right derivatives. Hence $a$ can be extremal for $t=1$ only if

or

$$
\|u\|_{\lambda}=\{(2+\underline{\sigma}) \psi(u)\}^{-1 / \underline{g}}>1
$$

$$
\|u\|_{\lambda}=\{(2+\bar{\sigma}) \psi(u)\}^{-1 / \bar{\sigma}}<1 .
$$


In the case where $\underline{\sigma}=\bar{\sigma}=: \sigma$, both conditions reduce to

$$
1=(2+\sigma) \psi(u)\|u\|_{\lambda}^{\sigma} \text {. }
$$

We put

where

$$
M_{\lambda}:=M_{\lambda}^{(i)} \cup M_{\lambda}^{(i i)}
$$

$$
\begin{aligned}
& M_{\lambda}^{(i)}:=\left\{u \in H_{T} \backslash\{0\}:\|u\|_{\lambda}=\{(2+\boldsymbol{\sigma}) \psi(u)\}^{-1 / \sigma}>1\right\}, \\
& M^{(i i)}:=\left\{u \in H_{T} \backslash\{0\}:\|u\|_{\lambda}=\{(2+\bar{\sigma}) \psi(u)\}^{-1 / \tilde{\sigma}}<1\right\} .
\end{aligned}
$$

(In the case where $g=\bar{\sigma}=\sigma$, simply set

$$
M_{\lambda}:=\left\{u \in H_{T} \backslash\{0\}: 1=(2+\sigma) \psi(u)\|u\|_{\lambda}^{\sigma} .\right)
$$

The two following lemmas give the central properties of these sets.

LEMMA 3.2. Let $u \in H_{T} \backslash\{0\}$ be fixed. If $I_{\lambda}(t u)$ is maximal for $t=1$ then $u \in M_{\lambda}$.

LEMMA 3.3.

(i) If $u \in M_{\lambda}^{(i)}$, then $I_{\lambda}(u)=\underline{\sigma} /(4+2 \sigma)\|u\| \|_{\lambda}^{2}>\sigma /(4+2 \sigma)$.

(ii) If $u \in M_{\lambda}^{(i i)}$, then $I_{\lambda}(u)=\bar{\sigma} /(4+2 \bar{\sigma})\|u\|_{\lambda}^{2}<\bar{\sigma} /(4+2 \bar{\sigma})$.

(iii) If $\bar{\sigma}=g=\sigma$ and $u \in M_{\lambda}$, then $I_{\lambda}(u)=\sigma /(4+2 \sigma)\|u\| \|_{\lambda}^{2}$.

As mentioned above, we investigate the critical points of $J_{\lambda}$ via a theorem of Ljusternik-Schirelman type given by Ambrosetti and Rabinowitz; $\left(I_{1}\right)-\left(I_{5}\right)$ will therefore refer to conditions on $J_{\lambda}$ given by these authors in [1]. We now show that $J_{\lambda}$ in fact satisfies these conditions if the following assumption is made on $f$.

(A3*) There exists $q>2$ such that for all $s \in \mathbb{R}$ and almost all $x \in \mathbb{R}^{N}$

$$
f(x, s) s \geqq q \mathscr{F}(x, s) \geqq 0 .
$$

Note that $f$ satisfies this condition if $f$ is of the form given in (3.1); simply set $q:=2+\underline{g}$. Assumption $\left(\mathrm{A} 3^{*}\right)$ means that

$$
\langle F(u), u\rangle \geqq q \varphi(u) \geqq 0 \text { for all } u \in H_{\mathrm{T}} ;
$$

Assumptions $\left(\mathrm{A} 2^{*}\right)$ and $\left(\mathrm{A} 3^{*}\right)$ together give that

$$
\langle F(u), u\rangle \geqq 2 \varphi(u)>0 \text { for all } u \in H_{T} \backslash\{0\} .
$$

We now suppose that $f$ satisfies Conditions $\left(\mathrm{A} 1^{*}\right),\left(\mathrm{A} 2^{*}\right)$ and $\left(\mathrm{A} 3^{*}\right)$ and show that $J_{\lambda}$ satisfies Conditions $\left(I_{1}\right)-\left(I_{5}\right)$ in [1] .

( $\left.\mathrm{I}_{1}\right)$ There exists $\rho, \alpha>0$ such that $J_{\lambda}>0$ on $B_{\rho} \backslash\{0\}$ and $J_{\lambda} \geqq \alpha>0$ on $S_{\rho}$ where $B_{\rho}:=\left\{u \in H_{T}:\|u\|_{\lambda}<\rho\right\}$ and $S_{\rho}:=\partial B_{\rho}$.

Proof. The proof can be found in [5]. For completeness, we just recall that, for $u \in H_{\mathrm{T}}$,

$$
J_{\lambda}(u) \geqq \frac{1}{2}\|u\|_{\lambda}^{2}-\frac{1}{2} \sum_{i=1}^{m} K_{i}\|T u\|^{\alpha_{i}}\|u\|^{\beta_{i}}
$$


by (3.2) and Proposition 2.1. Hence,

$$
J_{\lambda}(u) \geqq \frac{1}{2}\|u\|_{\lambda}^{2}\left[1-\max _{1 \leq i \leq m}\left\{m K_{i}\|u\|_{\lambda^{i}}^{\alpha_{i}+\beta_{i}-2}|\lambda|^{-\beta_{i} / 2}\right\}\right]
$$

and, since $\alpha_{i}+\beta_{i}>2$, the proof is complete.

$\left(\mathrm{I}_{2}\right) \quad$ There exists $e \in H_{\mathrm{T}} \backslash\{0\}$ such that $J_{\lambda}(e)=0$.

Proof. This follows immediately from $\left(I_{1}\right)$ and $\left(I_{5}\right)$ (see below). In fact there exist infinitely many such elements.

$\left(\mathrm{I}_{3}\right)$ If $\left\{u_{n}\right\}$ is a sequence in $H_{T}$ such that

$$
0<J_{\lambda}\left(u_{n}\right) \leqq \sup J_{\lambda}\left(u_{n}\right)<\infty
$$

and

$$
\left\|J^{\prime}\left(u_{n}\right)\right\|_{\left(H_{T}\right)^{*}} \rightarrow 0 \text { for } n \rightarrow \infty,
$$

then there exists a subsequence $\left\{u_{n^{\prime}}\right\}$ such that $u_{n^{\prime}}$ converges in $H_{T}$ to some $\bar{u}$.

Proof. For a proof see [5].

Remark. Condition $\left(\mathrm{I}_{3}\right)$ is the Palais-Smale condition (PS) ${ }^{+}$.

( $\left.\mathrm{I}_{4}\right) \quad J_{\lambda}$ is even: $J_{\lambda}(u)=J_{\lambda}(-u)$ for all $u \in H_{\mathrm{T}}$.

Proof. By $\left(\mathrm{A} 1^{*}\right), \varphi$ is an even functional. Therefore $J_{\lambda}$ is also even.

( $\left.\mathrm{I}_{5}\right)$ For any finite dimensional subspace $Z$ of $H_{T}$, the set $Z \cap\left\{u \in H_{T}: J_{\lambda}(u) \geqq 0\right\}$ is bounded.

Proof. For a proof see [5].

\section{The existence of infinitely many solutions}

According to $[\mathbf{1}]$, we set

$$
\Gamma:=\left\{g \in C\left([0,1], H_{T}\right): g(0)=0 \quad \text { and } \quad g(e)=1\right\}
$$

where $e$ is the element whose existence is given by $\left(\mathrm{I}_{2}\right)$; $\Gamma_{*}:=\left\{h \in C\left(H_{T}, H_{T}\right): h(0)=0\right.$,

where $h$ is a homeomorphism from $H_{\mathrm{T}}$ to $H_{\mathrm{T}}$ and $\left.h(B) \subset \hat{A}_{0}\right\}$

$$
\begin{gathered}
B:=\left\{u \in H_{\mathrm{T}}:\|u\|_{\lambda}<1\right\} \text { and } \hat{A}_{0}:=\left\{u \in H_{\mathrm{T}}: J_{\lambda}(u) \geqq 0\right\} ; \\
\Gamma^{*}:=\left\{h \in \Gamma_{*}: h \text { is odd }\right\} ; \\
\Gamma_{k}:=\left\{K \subset H_{\mathrm{T}}: K \text { is compact in } H_{\mathrm{T}}, K \text { is a symmetric set i.e. }-K=K,\right. \\
\left.\gamma(K \cap h(\partial B)) \geqq k, \quad \forall h \in \Gamma^{*} \quad(k \in \mathbb{N})\right\}
\end{gathered}
$$

where $\gamma$ is the genus of a set

If Conditions $\left(\mathrm{A} 1^{*}\right),\left(\mathrm{A} 2^{*}\right)$ and $\left(\mathrm{A} 3^{*}\right)$ are satisfied, equation (2.1) has for each $\lambda<0$ infinitely many (generalised) solutions corresponding to the critical values $b^{\lambda}$ 
and $b_{k}^{\lambda}(k \in \mathbb{N})$, where

$$
\begin{aligned}
b^{\lambda} & :=\inf _{\mathbf{g} \in \Gamma} \max _{u \in g([0,1])} J_{\lambda}(u), \\
b_{k}^{\lambda} & :=\inf _{K \in \Gamma_{k}} \max _{u \in K} J_{\lambda}(u) .
\end{aligned}
$$

\section{Behaviour of the solutions as $\boldsymbol{\lambda} \rightarrow 0^{-}$}

We discuss the behaviour of the solutions $u_{k}^{\lambda}$ to equation (2.1) which correspond to the critical values $b_{k}^{\lambda}$ when $\lambda \rightarrow 0$. Accordingly, we make the following assumption on $f$ :

(A4*) There exist constants $A, \delta, t>0$ such that

$$
\mathscr{F}(x, s) \geqq A(1+|x|)^{-t}|s|^{2+\bar{\sigma}}
$$

for all $|s|<\delta$ and for almost all $x \in \mathbb{R}^{N}$ where $\delta$ and $t$ satisfy the inequalities

$$
0<\delta<1 \text { and } 0<t<2+\underline{\sigma}-\bar{\sigma}-N \bar{\sigma} / 2,
$$

where $\bar{\sigma}$ and $g$ are the constants of Condition (A2*).

Note that Condition ( $4^{*}$ ) is satisfied by a function $f$ of the form (3.1) if

$$
q_{i}(x) \geqq A(1+|x|)^{-t} \quad \text { for almost all } x \in \mathbb{R}^{N} \quad(i=1 \ldots m) .
$$

What we want to show is that under Assumptions $\left(\mathrm{A} 1^{*}\right)-\left(\mathrm{A} 4^{*}\right)$ bifurcation will occur at the point $\lambda=0$.

Let $p(t):=\sum_{i=0}^{k-1} a_{i} t^{i}$ be a polynomial of degree $\leqq k-1$. We often identify $p(t)$ and $p:=\left(a_{0}, a_{1}, \ldots, a_{k-1}\right) \in \mathbb{R}^{k}$. The space $\mathbb{R}^{k}$ will be considered to be equipped with the norm

$$
\|p\|_{k}:=\max \left\{\left|a_{i}\right|: i=0, \ldots, k-1\right\}, \quad \forall p=\left(a_{0}, \ldots, a_{k-1}\right) \in \mathbb{R}^{k} ;
$$

this norm is equivalent to the usual one

$$
\|p\|:=\left\{\sum_{i=0}^{k-1} a_{i}^{2}\right\}^{\frac{1}{2}}, \quad \forall p=\left(a_{0}, \ldots, a_{k-1}\right) \in \mathbb{R}^{k} .
$$

For $p \in \mathbb{R}^{k}$, we set

$$
\begin{aligned}
d_{p} & :=\left\{\int p^{2}\left(|y|^{2}\right) e^{-2|y|^{2}} d y\right\}^{\frac{1}{2}}, \\
L_{p} & :=4 \int|y|^{2}\left[p^{\prime}\left(|y|^{2}\right)-p\left(|y|^{2}\right)\right]^{2} e^{-2|y|^{2}} d y, \\
K_{p} & :=2^{-t} A \int_{|y| \geqq 1}|y|^{-t}\left|p\left(|y|^{2}\right)\right|^{2+\bar{\sigma}_{e}} e^{-(2+\bar{\sigma})|y|^{2}} d y .
\end{aligned}
$$

We first give some properties of $d_{\mathrm{p}}, L_{\mathrm{p}}$ and $K_{\mathrm{p}}$.

LEMMA 5.1. $d_{p}$ depends continuously on $p$, for all $p \in \mathbb{R}^{k}$. 
Proof. Let

Then

$$
\begin{aligned}
p & :=\left(a_{0}, \ldots, a_{k-1}\right) \in \mathbb{R}^{k}, \\
\varepsilon & :=\left(\varepsilon_{0}, \ldots, \varepsilon_{k-1}\right) \in \mathbb{R}^{k}, \\
p+\varepsilon: & =\left(a_{0}+\varepsilon_{0}, \ldots, a_{k-1}+\varepsilon_{k-1}\right) \in \mathbb{R}^{k} .
\end{aligned}
$$

$$
\begin{aligned}
d_{\mathrm{p}+\varepsilon}^{2}-d_{\mathrm{p}}^{2}= & 2 \int \varepsilon\left(|y|^{2}\right) p\left(|y|^{2}\right) e^{-2|y|^{2}} d y+\int \varepsilon^{2}\left(|y|^{2}\right) e^{-2|y|^{2}} d y \\
= & \sum_{i=0}^{2 k-2} \sum_{j=0}^{i}\left\{2 \int \varepsilon_{j} a_{i-j}|y|^{2 i} e^{-2|y|^{2}} d y\right. \\
& \left.+\int \varepsilon_{j} \varepsilon_{i-j}|y|^{2 i} e^{-2|y|^{2}} d y\right\} \\
= & \sum_{i=0}^{2 k-2} \sum_{j=0}^{i} \varepsilon_{j}\left(2 a_{i-j}+\varepsilon_{i-j}\right) \int|y|^{2 i} e^{-2|y|^{2}} d y,
\end{aligned}
$$

where $a_{s}=\varepsilon_{s}=0$ for $s \geqq k$. Therefore,

$$
d_{\mathrm{p}+\varepsilon}^{2}-d_{\mathrm{p}}^{2} \rightarrow 0 \text { as }\|\varepsilon\|_{k} \rightarrow 0 .
$$

This proves the continuity of $d_{\mathrm{p}}$.

LEMMA 5.2. There exists a constant $\mathscr{L}>0$ such that

$$
0 \leqq L_{p} \leqq \mathscr{L}\|p\|_{k}^{2}, \quad \forall p \in \mathbb{R}^{k} .
$$

Proof. Let $p=\left(a_{0}, \ldots, a_{k-1}\right) \in \mathbb{R}^{k}$. Then

$$
\begin{aligned}
0 \leqq L_{p} & =4 \int|y|^{2}\left[p^{\prime}\left(|y|^{2}\right)-p\left(|y|^{2}\right)\right]^{2} e^{-2|y|^{2}} d y \\
& =4 \int|y|^{2}\left\{\sum_{i=0}^{k-1}\left[(i+1) a_{i+1}-a_{i}\right]|y|^{2 i}\right\}^{2} e^{-2|y|^{2}} d y,
\end{aligned}
$$

where $a_{k}$ is taken to be zero. Since

$$
\begin{aligned}
\left|(i+1) a_{i+1}-a_{i}\right| & \leqq \\
& =(k+1) \max \left\{\left|a_{i}\right|: i=0, \ldots, k-1\right\} \\
& (k+1) \|_{k},
\end{aligned}
$$

we have

$$
0 \leqq L_{p} \leqq 4(k+1)^{2} \int|y|^{2}\left\{\sum_{i=0}^{k-1}|y|^{2 i}\right\}^{2} e^{-2|y|^{2}} d y \cdot\|p\|_{k}^{2}
$$

If we put

$$
\mathscr{L}:=4(k+1)^{2} \int|y|^{2}\left\{\sum_{i=0}^{k-1}|y|^{2 i}\right\}^{2} e^{-2|y|^{2}} d y,
$$

the lemma is proved.

LEMMA 5.3. (i) $K_{\mathrm{p}}$ depends continuously on $p$, for all $p \in \mathbb{R}^{k}$. (ii) $K_{\mathrm{p}}=0$ if and only if $p=0$. 
Proof. (i) Let $p:=\left(a_{0}, \ldots, a_{k-1}\right) \in \mathbb{R}^{k}$,

Then

$$
\varepsilon:=\left(\varepsilon_{0}, \ldots, \varepsilon_{k-1}\right) \in \mathbb{R}^{k} \text { with }\|\varepsilon\|_{k}<1 .
$$

$$
K_{p+\varepsilon}=2^{-t} A \int_{|y| \geqq 1}|y|^{-t}\left|(p+\varepsilon)\left(|y|^{2}\right)\right|^{2+\bar{\sigma}} e^{-(2+\bar{\sigma})|y|^{2}} d y .
$$

If one takes as dominating function

where

$$
y \mapsto|y|^{-t}\left|\tilde{p}\left(|y|^{2}\right)\right|^{2+\bar{\sigma}} e^{-(2+\bar{\sigma})|y|^{2}}, \quad y \in \mathbb{R}^{N},
$$

$$
\tilde{p}(t):=\sum_{i=0}^{k-1}\left(\left|a_{i}\right|+1\right) t^{i},
$$

the Lebesgue dominated convergence theorem gives

$$
K_{p+\varepsilon} \rightarrow K_{p} \quad \text { as }\|\varepsilon\|_{k} \rightarrow 0 .
$$

(ii) This follows from the definition of $K_{\mathrm{p}}$.

For $p \in \mathbb{R}^{k}$ and $\lambda<0$, we put

$$
u_{p, \lambda}(x):=p\left(-\lambda|x|^{2}\right) e^{\lambda|x|^{2}}, \quad x \in \mathbb{R}^{N},
$$

and we consider the following subspace

$$
Z(k, \lambda):=\left\{u_{\mathrm{p}, \lambda}(x): p \in \mathbb{R}^{k}\right\} \subset H_{\mathrm{T}} .
$$

By Condition $\left(I_{5}\right)$, the set

$$
Z(k, \lambda)_{+}:=Z(k, \lambda) \cap\left\{u \in H_{T}: J_{\lambda}(u) \geqq 0\right\}
$$

is bounded in $H_{T}$ and hence in $Z(k, \lambda)$. The following proposition shows that this boundedness is uniform if $|\lambda|$ is small enough.

Propositron 5.4. Let Conditions (A1*)-(A4*) hold and put

$$
\tilde{Z}(k, \lambda):=\left\{u_{\mathrm{p}, \lambda}(x) \in Z(k, \lambda):\|p\|_{k} \leqq 1\right\} .
$$

Then there exists a constant $\lambda_{0} \in[-1,0)$ such that

$$
Z(k, \lambda)_{+} \subset \tilde{Z}(k, \lambda) \text { whenever } \lambda \in\left(\lambda_{0}, 0\right) .
$$

Proof. Let $Z:=\left\{u_{\mathrm{p}, \lambda}(x) \in Z(k, \lambda):\|p\|_{k}=1\right\}$. For the majorant functional $I_{\lambda}$ we show that

$$
\left.I_{\lambda}\right|_{Z}(u)<0 \text { whenever } \lambda \in\left(\lambda_{0}, 0\right) .
$$

The conclusion of the proof follows from the radial behaviour of $I_{\lambda}$ and the connection between $J_{\lambda}$ and $I_{\lambda}$.

We first remark that

and

$$
\begin{aligned}
\left\|u_{p, \lambda}\right\|^{2} & =|\lambda|^{-N / 2} d_{p}^{2}, \\
\left\|T u_{p, \lambda}\right\|^{2} & =|\lambda|^{1-N / 2} L_{p},
\end{aligned}
$$

$$
\left\|u_{\mathrm{p}, \lambda}\right\|_{\lambda}^{2}=|\lambda|^{1-\mathrm{N} / 2}\left(L_{\mathrm{p}}+d_{\mathrm{p}}^{2}\right) \quad \text { for all } \quad u_{\mathrm{p}, \lambda}(x) \in Z(k, \lambda) .
$$


These equalities can easily be verified by a direct computation. We now put

and

$$
y(p):=2 \max _{x \in \mathbb{R}^{N}}\left|u_{p, \lambda}(x)\right|, \quad \forall p \in \mathbb{R}^{k}
$$

$$
y_{0}:=\min \left\{y(p): p \in \mathbb{R}^{k} \quad \text { with }\|p\|_{k}=1\right\} .
$$

Note that $y(p)$ is always finite and depends continuously on $p$. Since $y(p)>0$ whenever $p \neq 0$, we thus have $y_{0}>0$. If $\delta$ is the constant in Condition $\left(\mathrm{A} 4^{*}\right)$, we have, by $\left(\mathrm{A} 2^{*}\right)$,

where

$$
\begin{aligned}
\varphi\left(u_{p, \lambda}\right) & =\varphi\left(\frac{y(p)}{\delta} \cdot \frac{\delta}{y(p)} u_{p, \lambda}\right) \\
& \geqq[y(p) / \delta]^{2+\sigma} \varphi\left(\frac{\delta}{y(p)} u_{p, \lambda}\right)
\end{aligned}
$$

$$
\sigma= \begin{cases}\bar{\sigma} & \text { if } y(p) / \delta<1 \\ \underline{\sigma} & \text { otherwise }\end{cases}
$$

Therefore, by $\left(\mathrm{A} 4^{*}\right)$,

$$
\begin{aligned}
\varphi\left(u_{p, \lambda}\right) & \geqq[y(p) / \delta]^{(2+\sigma) / 2+\bar{\sigma})} 2^{-t} A \int_{|x| \geqq 1}|x|^{-t}\left|p\left(\lambda|x|^{2}\right)\right|^{2+\bar{\sigma}} e^{\lambda(2+\bar{\sigma})|x|^{2}} d x \\
& \geqq\left.[y(p) / \delta]^{(2+\sigma) /(2+\bar{\sigma})} 2^{-t} A|\lambda|^{(t-N) / 2} \int_{|y| \geqq 1}|y|^{-t}\left|p\left(-|y|^{2}\right)^{2+\tilde{\sigma}^{-(2+\bar{\sigma})}}\right| y\right|^{2} d y
\end{aligned}
$$

if $|\lambda| \leqq 1$. Hence, for $|\lambda| \leqq 1$,

where

$$
\varphi\left(u_{\mathrm{p}, \lambda}\right) \geqq C \cdot K_{\mathrm{p}} \cdot|\lambda|^{(t-N) / 2}
$$

$$
C:=\min \left\{\left[y_{0} / \delta\right]^{(2+\sigma) /(2+\bar{\sigma})}, y_{0} / \delta\right\}>0 \text {. }
$$

If we set

$$
\begin{aligned}
& D:=\max \left\{L_{\mathrm{p}}+d_{p}^{2}: p \in \mathbb{R}^{k} \quad \text { with }\|p\|_{k}=1\right\}, \\
& K:=\min \left\{K_{\mathrm{p}}: p \in \mathbb{R}^{k} \quad \text { with }\|p\|_{k}=1\right\},
\end{aligned}
$$

we have, by Lemmas $5.1,5.2$ and 5.3, that $D$ and $K$ are finite and positive. Hence, for $\lambda \in[-1,0)$,

$$
\begin{aligned}
I_{\lambda}\left(u_{p, \lambda}\right) & \leqq \frac{1}{2}|\lambda|^{1-N / 2}\left(L_{p}+d_{p}^{2}\right)-C K_{\mathrm{p}}|\lambda|^{(t-N) / 2} \\
& \leqq|\lambda|^{(t-N) / 2}\left(\frac{1}{2}|\lambda|^{1-t / 2} D-C \cdot K\right) .
\end{aligned}
$$

Therefore, there exists some $\lambda_{0} \in[-1,0)$ such that

$$
I_{\lambda}\left(u_{p, \lambda}\right)<0 \text { for all } u_{p, \lambda}(x) \in Z
$$

where $\lambda \in\left(\lambda_{0}, 0\right)$.

Remark. By the proof of Lemma 2.7 in [1],

$$
\tilde{Z}(k, \lambda) \in \Gamma_{k}(k \in \mathbb{N}) \text { for } \lambda \in\left(\lambda_{0}, 0\right) .
$$


Proposition 5.5. Let Conditions $\left(\mathrm{A} 1^{*}\right)-\left(\mathrm{A} 4^{*}\right)$ hold and let $\lambda_{0}$ be given by Proposition 5.4. Then there exists a constant $\lambda_{1} \in\left[\lambda_{0}, 0\right)$ such that

$$
\psi\left(u_{\mathrm{p}, \lambda}\right) \geqq 2 \text { for all } u_{\mathrm{p}, \lambda} \in \tilde{Z}(k, \lambda) \backslash\{0\}
$$

where $\lambda \in\left(\lambda_{1}, 0\right)$.

Proof. For $u_{\mathrm{p}, \lambda} \in \tilde{Z}(k, \lambda) \backslash\{0\}$, we have

where

$$
\psi\left(u_{p, \lambda}\right)=\varphi\left(u_{p, \lambda}\|\| u_{p, \lambda} \|_{\lambda}\right) \geqq\left\|u_{p, \lambda}\right\| \|_{\lambda}^{-(2+\sigma)} \varphi\left(u_{p, \lambda}\right)
$$

Hence,

$$
\sigma= \begin{cases}\bar{\sigma} & \text { if }\left\|u_{\mathrm{p}, \lambda}\right\| \|_{\lambda} \geqq 1, \\ \underline{\sigma} & \text { otherwise }\end{cases}
$$

where

$$
\psi\left(u_{\mathrm{p}, \lambda}\right) \geqq\left\{|\lambda|^{1-N / 2}\left(L_{\mathrm{p}}+d_{\mathrm{p}}^{2}\right)\right\}^{-1-\sigma / 2} \cdot C_{\mathrm{p}} \cdot K_{p} \cdot|\lambda|^{(t-N) / 2}
$$

$$
C_{\mathrm{p}}:=\min \left\{[y(p) / \delta]^{(2+\boldsymbol{g}) /(2+\bar{\sigma})}, \quad y(p) / \delta\right\} .
$$

But since

$$
\psi\left(t u_{\mathrm{p}, \lambda}\right)=\psi\left(u_{\mathrm{p}, \lambda}\right) \text { for all } t>0
$$

we get

$$
\psi\left(u_{p, \lambda}\right) \geqq D^{-1-\sigma / 2} C K|\lambda|^{\kappa}
$$

where $C, D$ and $K$ are the same as in the proof of Proposition 5.4 and where $\kappa$ is given by

So

$$
\kappa=(N / 2-1)(1+\sigma / 2)+(t-N) / 2<-1+N \tilde{\sigma} / 4-\sigma / 2+t / 2<-\bar{\sigma} / 2<0 .
$$

$$
\psi\left(u_{\mathrm{p}, \lambda}\right) \rightarrow \infty \quad \text { for } \quad \lambda \rightarrow 0^{-} \quad \text { uniformly on } \quad \tilde{Z}(k, \lambda) \backslash\{0\} .
$$

Proposition 5.6. Let Conditions (A1*)-(A4*) hold. Let $u_{k}^{\lambda}$ be the (generalised) solution to (2.1) corresponding to the critical value $b_{k}^{\lambda}(\lambda<0, k \in \mathbb{N})$. Then

$$
b_{k}^{\lambda} \rightarrow 0 \text { as } \lambda \rightarrow 0^{-} \text {. }
$$

More precisely,

$$
b_{k}^{\lambda}=o(\lambda) \text { for } \lambda \rightarrow 0^{-} .
$$

Proof. Suppose that $\underline{\sigma}<\bar{\sigma}$. For $\underline{\sigma}=\bar{\sigma}$, the proof remains the same except that $M_{\lambda}^{(i i)}$ is replaced by $M_{\lambda}$. Suppose $\lambda \in\left(\lambda_{1}, 0\right)$. Then

$$
\begin{aligned}
0<b_{k}^{\lambda} & =\inf _{K \in \Gamma_{k}} \max _{u \in K} J_{\lambda}(u) \\
& \leqq \max _{u \in \tilde{Z}(k, \lambda)} I_{\lambda}(u) \\
& \leqq \max _{u \in \tilde{Z}(k, \lambda) \cap M_{\lambda}^{(i)}} I_{\lambda}(u)
\end{aligned}
$$

since $\tilde{Z}(k, \lambda) \cap M_{\lambda}^{(i)}=\varnothing$ by Proposition 5.5. If we put

$$
t(u):=[(2+\bar{\sigma}) \psi(u)]^{-1 / \bar{\sigma}}\left(L_{p}+d_{p}^{2}\right)^{-\frac{1}{2}}|\lambda|^{-\frac{1}{2}+N / 4}
$$


and

then

$$
Z_{\lambda}:=\left\{u_{p, \lambda} \in Z(k, \lambda):\|p\|_{k}=1\right\}
$$

$$
t\left(u_{\mathrm{p}, \lambda}\right) u_{\mathrm{p}, \lambda} \in M_{\lambda}^{(i i)} \quad \text { whenever } \quad u_{\mathrm{p}, \lambda} \in Z_{\lambda}
$$

and so

$$
\begin{aligned}
0<b_{k}^{\lambda} & \leqq \max _{u \in Z_{\lambda}} I_{\lambda}(t(u) u) \\
& =\bar{\sigma} /(4+2 \bar{\sigma}) \max _{u \in Z_{\lambda}}\|t(u) u\|_{\lambda}^{2} \\
& =\bar{\sigma} /(4+2 \bar{\sigma}) \max _{u \in Z_{\lambda}}[(2+\bar{\sigma}) \psi(u)]^{-2 / \bar{\sigma}} \\
& \leqq \text { const }|\lambda|^{-2 \kappa / \bar{\sigma}}
\end{aligned}
$$

by the proof of Proposition 5.5. Since $-2 \kappa / \bar{\sigma}>1$, we have

$$
b_{k}^{\lambda}=o(\lambda) \text { for } \lambda \rightarrow 0^{-} \text {. }
$$

We are now ready to prove the main theorem.

THEOREM 5.7. Let Conditions $\left(\mathrm{A} 1^{*}\right)-\left(\mathrm{A} 4^{*}\right)$ hold. Then (i) for $\lambda<0$, equation (2.1) has infinitely many distinct pairs of (generalised) solutions $\left\{\left(\lambda, \pm u_{k}^{\lambda}\right)\right\}_{k \in \mathbb{N}}$; (ii) $\lambda=0$ is a bifurcation point for equation (2.1), i.e.

$$
\left\|u_{k}^{\lambda}\right\|_{T} \rightarrow 0 \text { as } \lambda \rightarrow 0^{-} \text {. }
$$

Proof. (i) This is a result of Section 4.

(ii) Let $u_{k}^{\lambda}$ be a critical point of $J_{\lambda}$ :

$$
J_{\lambda}\left(u_{k}^{\lambda}\right)=b_{k}^{\lambda}, \quad J_{\lambda}^{\prime}\left(u_{k}^{\lambda}\right)=0 .
$$

Therefore,

$$
\left.\frac{d}{d t} J_{\lambda}\left(t u_{k}^{\lambda}\right)\right|_{t=1}=0, \quad \text { i.e. } \quad\left\|u_{k}^{\lambda}\right\| \|_{\lambda}^{2}=\left\langle F\left(u_{k}^{\lambda}\right), u_{k}^{\lambda}\right\rangle
$$

But

$$
\begin{aligned}
b_{k}^{\lambda} & =\frac{1}{2}\left\|u_{k}^{\lambda}\right\|_{\lambda}^{2}-\varphi\left(u_{k}^{\lambda}\right) \\
& =\frac{1}{2}\left\langle F\left(u_{k}^{\lambda}\right), u_{k}^{\lambda}\right\rangle-\varphi\left(u_{k}^{\lambda}\right)=o(\lambda) \text { for } \lambda \rightarrow 0
\end{aligned}
$$

and thus by $\left(\mathrm{A} 3^{*}\right)$

$$
b_{k}^{\lambda} \geqq\left(\frac{1}{2} q-1\right) \varphi\left(u_{k}^{\lambda}\right) \geqq 0 \rightarrow \varphi\left(u_{k}^{\lambda}\right)=o(\lambda) \text { for } \lambda \rightarrow 0^{-} .
$$

Therefore,

i.e.

$$
\left\langle F\left(u_{k}^{\lambda}\right), u_{k}^{\lambda}\right\rangle=o(\lambda) \text { for } \lambda \rightarrow 0^{-},
$$

$$
\left\|u_{k}^{\lambda}\right\|_{\lambda}^{2}=o(\lambda) \text { for } \lambda \rightarrow 0^{-} .
$$

Since $\|u\|_{\lambda} \geqq\|T u\|$ and $\|u\|_{\lambda} \geqq \sqrt{|\lambda|}\|u\|$ for all $u \in H_{T}$, we have

$$
\begin{aligned}
\left\|T u_{k}\right\|^{2} & =o(\lambda), \\
\left\|u_{k}\right\|^{2} & =o(1), \\
\left\|u_{k}\right\|_{T}^{2} & =o(1) \text { for } \lambda \rightarrow 0^{-} .
\end{aligned}
$$




\section{An alternative approach}

There is another approach to the problem (2.1) given by Stuart [4].

We put

and

$$
J(u):=\frac{1}{2}\|T u\|^{2}-\varphi(u) \text { for } u \in H_{T}
$$

$$
M_{r}:=\left\{u \in H_{T}:\|u\|=r\right\} \text { for } r>0 .
$$

Then critical points of $\left.J\right|_{M_{r}}$ are (generalised) solutions to (2.1) and it is sufficient to verify the hypotheses of Theorem 4 of [5]. The main point is to show that Assumption (S4) is satisfied for all $j \in \mathbb{N}$ and this can be done (under the assumptions on $f$ given below) using functions of type (1.1) and calculations similar to those of Section 5. The details will appear in [7].

Let us make the following (weaker) assumptions on $f$ :

(A2) $\mathscr{F}(x, t s) \geqq t^{2} \mathscr{F}(x, s) \geqq 0$ for all $s \in \mathbb{R}$ all $t \geqq 1$ and almost all $x \in \mathbb{R}^{N}$.

(A3) $f(x, s) s \geqq 2 \mathscr{F}(x, s) \geqq 0$ for all $s \in \mathbb{R}$ and almost all $x \in \mathbb{R}^{N}$.

(A4) There exist positive constants $\sigma, \delta, A$ and $t$ such that $0<\sigma<2(2-t) / N$ and $\mathscr{F}(x, s) \geqq A(1+|x|)^{-t}|s|^{2+\sigma}$ for all $0 \leqq s \leqq \delta$ and almost all $x \in \mathbb{R}^{N}$.

THEOREM 6.1. Let Conditions (A1*), (A2)-(A4) hold. Then, for $r>0$ small enough, there exist infinitely many distinct pairs of (generalised) solutions $\left(\lambda_{n}^{r}\right.$, $\left.\pm u_{n}^{r}\right) \in \mathbb{R} \times H_{\mathrm{T}}$ for equation (2.1) such that

$$
\begin{array}{cll}
\left\|u_{n}^{r}\right\|=r, & \lambda_{n}^{r}<0 & \text { for all } n \in \mathbb{N} . \\
\left\|u_{n}^{r}\right\|_{T} \rightarrow 0, & \lambda_{n}^{r} \rightarrow 0^{-} & \text {as } r \rightarrow 0^{+} .
\end{array}
$$

Let us remark that the question as to whether Condition $\left(\mathrm{A} 1^{*}\right)$ can be weakened remains open.

\section{References}

1 A. Ambrosetti and P. H. Rabinowitz. Dual variational methods in critical point theory and applications. J. Funct. Anal. 14 (1973), 359-381.

2 H. Berestycki and P.-L. Lions. Nonlinear scalar field equations I (Existence of a ground state) and II (Existence of infinitely many solutions). Univ. Paris VI preprints. Arch. Rational Mech. Anal. to appear.

3 P. H. Rabinowitz. Variational methods for nonlinear eigenvalue problems, C.I.M.E., ed. Prodi, G. (Rome: Ed. Cremonese, 1974).

4 C. A. Stuart. Bifurcation from the continuous spectrum in the $L^{2}$-theory of elliptic equations on $\boldsymbol{R}^{\mathbf{N}}$. Recent Methods in Nonlinear Analysis and Applications, Lignori, Napoli (1981) (copies available from the author).

5 C. A. Stuart. Bifurcation from the essential spectrum. Proceedings of Equadiff 82. Lecture Notes in Mathematics 1017 (Berlin: Springer, 1983).

6 E. Zeidler. Vorlesungen über nichtlineare Funktionalanalysis III-Variationsmethoden und Optimierung (Teubner Texte zur Mathematik) (Leipzig: Teubner, 1977).

7 H.-J. Ruppen. Le Probleme de Dirichlet: Existence d'un nombre infini de branches de bifurcation pour $N \geqq 2$; existence et nonexistence de solutions pour $N=1$ (Thesis, EPF Lausanne, to appear).

(Issued 12 December 1985) 